\title{
Negative Energy Condition and Black Holes on the Brane
}

\author{
Naresh Dadhich \\ IUCAA, Post Bag 4, Ganeshkhind, Pune 411007, India.
}

\begin{abstract}
We propose that for non-localizable energy distribution the relevant energy condition is determined by the gravitational field energy which is negative for positive non-gravitational energy. That is negativity of the non-localized energy is the "positive" energy condition. This would have direct application and relevance for a black hole on the brane which would be sitting in a trace free stresses induced by the Weyl curvature of the bulk.
\end{abstract}

PACS Numbers: 04.50.+h, 04.60., 04.70.-s, 98.80.Cq

In view of the developments in the string theory, the study of gravity in dimensions higher than the usual four has been in vogue for quite a while. The recent results indicate that extra dimensions need not be compact, they could be large and even infinite $[1,2]$. In the Randall - Sundrum (R - S) model [2], the 4-D Universe is a 3-brane acting as a domain wall between the two semi infinite 5 -D negative $\lambda$ regions. That is, the actual Universe is embedded in the 5 -D bulk satisfying the appropriate boundary conditions. All non-gravitational matter fields remain localized in the brane while free gravitational field and gravitational waves also propagate off the brane in the 5-D bulk. The free field in the bulk backreacts on the brane to induce a trace free stress tensor through the 5-D Weyl tensor evaluated at the brane. The Einstein field equation for the brane has been formulated by Shiromizu, Maeda and Sasaki [3].

In the absence of energy distribution on the brane, the stress tensor on the brane would have only the trace free Weyl term which need not a priori satisfy the usual positive energy conditions. The energy density of the induced stresses could as well be negative. The effective stress tensor on the brane could thus legitimately include negative energy. It turns out that for a static black hole on the brane, the induced energy from the free gravitational field in the bulk must be negative to contribute positively to the hole's gravity (attractively). In this context we would like to argue that the proper energy condition should be that the induced energy must be negative to contribute 
attractively in unison with the hole. This should be true in general but we shall establish this explicitly for a static source.

The basic criterion for energy conditions is that they should ensure the usual behaviour for matter which we observe in the real life experience directly or indirectly. For instance, gravitational field produced by any kind of energy distribution must be attractive is one such condition. This is the case for non-gravitational energy distribution. However it translates for the gravitational field energy that it is negative. The field energy is non-localizable so could also be non-gravitational energy distribution like the electric field energy. Now the question is, what norm should be applicable to such a distribution; positive with the non-gravitational energy or negative with the field energy? We would argue that the latter is the case and would define the proper energy condition for non-localizable energy is that it be negative. That is, it would contribute attractively only when it is negative. That is, positive energy is repulsive.

This has direct relevance and application for the induced energy on the brane from the bulk. It has zero trace and hence it cannot be localized. It must follow the norm of the field energy. It must therefore be negative to produce attractive gravity on the brane. This will in turn determine the sign of the "tidal" charge.

Let me first give a couple of known examples of positive energy producing repulsive effect. Both for the charged and the Schwarzschild - de Sitter black holes, the electric field as well as the cosmological constant produce positive energy density, which produces repulsive gravity. It should not therefore come as surprise, however such an explicit statement is perhaps being made for the first time. Very recently, Vollick [4] has argued that energy density induced by the 5 -D vacuum solution onto the brane would be negative. Negative energy has been considered in quantum field theory for long time [5]. It has also been argued that it should also satisfy certain inequalities [6] restricting the duration of occurrence when observed. It turns out that more negative the density is shorter would be its life time.

Consider a simple but intuitively and physically illuminating situation. Let an isolated particle of mass $M$ sit in a non-gravitational energy distribution which falls off to zero asymptotically. That is, the total energy at infinity is $M$, while the energy contained inside some radius $R$ would be $M-E(r>R)$. In this, the energy lying exterior to $R$ has been subtracted out from the energy at infinity, $E(\infty)=M$. The Newtonian potential would then be given by,

$$
\phi(R)=-\frac{M-E(r>R)}{R}
$$

and the acceleration would be

$$
a=-\phi^{\prime}=-\frac{M-E}{R^{2}}-\frac{E^{\prime}}{R} .
$$

Since $E$ decreases with $r$, the second term would be positive implying 
attractive gravity in unison with the mass point. This clearly illustrates the important point that energy distribution engulfing the particle would act attractively in unison only when it is negative. We could apply this argument straightaway to the Reissner - Nordström solution of a charged black hole [7]. It is quite illuminating to see that the electric field energy would produce repulsive effect.

We shall now establish this result for a static source in general relativity (GR) and also formulate the proper energy condition for non-localized energy. In GR non-gravitational matter/energy as well as the gravitational field energy has gravitational charge. The difference in their action is that the former imparts a pull through the gradient of potential on the test particle while the latter warps/curves the space around the gravitating body [8]. However the two must work in resonance. That is the space warping must be in line with the pull.

For a static source, the spacetime metric is given by

$$
d s^{2}=-A d t^{2}+B d r^{2}+r^{2}\left(d \theta^{2}+\sin ^{2} \theta d \phi^{2}\right)
$$

where $A$ and $B$ are functions of the radial coordinate $r$. The gravitational potential sits in $A$ and imparts gravitational attraction on test particle which is given by $-A^{\prime} / 2 A=-M / r^{2}$. On the other hand the space curvature has the following non-zero Riemann components,

$$
R_{r \theta}^{r \theta}=R_{r \phi}^{r \phi}=\frac{B^{\prime}}{2 A B r}=-M / r^{3}, \quad R_{\theta \phi}^{\theta \phi}=\frac{1-1 / B}{r^{2}}=2 M / r^{3}
$$

where the terms with $M$ refer to the Schwarzschild solution.

From the Einstein equation, we can write for the energy density,

$$
\rho=\frac{B^{\prime}}{B^{2} r}+\frac{1}{r^{2}}\left(1-\frac{1}{B}\right)
$$

Note that the 3-space has the topology of $S^{2} X R$. From the point of view of radial motion, we can specialize to the equatorial plane. The metric for the Schwarzschild case, $A=B^{-1}=1-2 M / r$, would read as

$$
(1-2 M / r)^{-1} d r^{2}+r^{2} d \phi^{2}
$$

which has the negative curvature $-M / r^{3}$. It can be embedded in the 3 -flat space by writing

$$
z^{2}=8 M r-16 M^{2}
$$

which is a parabola and would generate a paraboloid of revolution (see Fig.1). Clearly it has negative curvature which would tend particles to roll down (or oppose motion leading to increasing $r$ ) to decreasing value of $r$ in unison with the attractive action of the potential. That is negative curvature here is the analogue of the attraction in the equation of motion.

Thus the norm for the curvature is set that it should be negative. The 
to the curvature and consequently positively (in line with the potential) to the field. The positive density would contribute positively to the curvature, opposing the potential, thereby weakening the field though overall curvature may still be negative as is the case for the charged black hole.

Corresponding to the positive energy conditions for the matter fields, the "positive" energy condition for the non-localized energy distribution in line with the gravitational field energy is that it be negative. We have used the prototype Schwarzschild field to ellucidate the norm set by gravitational field energy. The positivity of energy could be equivalently be stated as negativity of the field energy which is further equivalent to negativity of space curvature in $(r, \theta)$ plane.

This alternative formulation of the energy condition is particularly pertinent to the $\mathrm{R}$ - S brane world scenario [2]. Here, the free gravitational filed reflected from the bulk onto the brane through the Weyl curvature of the bulk would give rise to trace free stress tensor on the brane. It would represent a non-localizable energy distribution and hence the only energy condition which is pertinent in this case is the one we have formulated above. That is, it be negative to warp/curve the space in harmony with the action of the gravitational potential.

This has direct relevance and application for black hole on the brane, which would always have some non-zero energy distribution around it coming from the bulk Weyl curvature. It is therefore important that the induced energy density must be negative if it were to contribute positively (attractively) to the hole's field. This should really be the case because only the free field propagates in the bulk which would have negative energy and consequently it would reflect back negative energy density on the brane. Since the induced stresses are trace free, the energy distribution is non-localizable and hence it should follow the negativity norm of the gravitational field energy. Recently, a solution for a black hole on the brane has been proposed [9] which is described by the 4-D Reissner-Nordström metric for the charged black hole. Of course there is no electric charge, the corresponding parameter refers to the "tidal" charge induced by the bulk Weyl curvature. This charge parameter would, in contrast to the electric charge case, be negative because the gravitational field energy in the bulk is negative and to satisfy the proper negative energy condition. This solution would be valid only near the horizon. The high energy corrections to the Schwarzschild solution should only introduce modification to the field without altering its basic character. The "tidal" charge would therefore have to be negative. If it is not so, it would give rise to both the event as well as the Cauchy horizon which will change the singularity character of the static black hole. What is expected is a modification retaining the basic character of the spacetime. Thus the tidal charge parameter and the energy of the distribution immersing the hole must be negative.

All this discussion of negativity of energy would have direct application to the study of gravitational collapse on the brane. As the high energy 
It would therefore act in unison with the gravitational attraction and would strengthen the collapse. They would act in line with the cosmic censorship hypothesis for collapse on the brane. It would be interesting to investigate the cases on the brane that lead to a naked singularity in GR [10]. Would the singularity still persist or not? The answer to this question would be of significance for the ultimate outcome of gravitational collapse.

Acknowledgements: This work was started at the Albert Einstein Institute, Golm and carried through to the universities of Wales, Cardiff, of the Basque Country, Bilbao and of Portsmouth, and Queen Mary and Westfield College, London. I wish to thank all these institutions and my hosts for warm hospitality. I would also like to thank T. Shiromizu, Roy Maartens and Sanjeev Dhurandhar for useful discussions.

\section{References}

[1] N. Arkani-Hamed, S. Dimopoulos and G. Dvali, Phys. Lett. B429, (1998) 263, hep-ph/9803315; I. Antoniadis, N. Arkani-Hamed, S. Dimpoulos and G. Dvali, Phys. Lett. B436, (1998) 257, hep-ph/9804398.

[2] L. Randall and R. Sundrum, Phys. Rev. Lett. 83, (1999) 3370 and 4690, hep-ph/9906064.

[3] T. Shiromizu, K. Maeda and M. Sasaki, gr-qc/9910076; M. Sasaki, T. Shiromizu and K. Maeda, hep-th/9912233.

[4] D. N. Vollick, hep-th/0004064.

[5] H. Epstein, V. Glaser and A. Jaffe, Nouvo Cimento 36, (1965) 1016.

[6] L. H. Ford and T. A. Roman, Phys. Rev. D55,(1997) 2082; L. H. Ford, Phys. Rev. D43, (1991) 3972.

[7] N. Dadhich, in A Random Walk in Relativity and Cosmology, eds. N. Dadhich et al, (Wiley Eastern Ltd.,1985) p.72.

[8] N. Dadhich, gr-qc/9704068.

[9] N. Dadhich, R. Maartens, P. Papadopoulos and V. Rezania, Phys. Lett. B487,(2000) 1, hep-th/0003061.

[10] P. S. Joshi, Global Aspects in Gravitation and Cosmology, Clarendon, Oxford, 1993. 


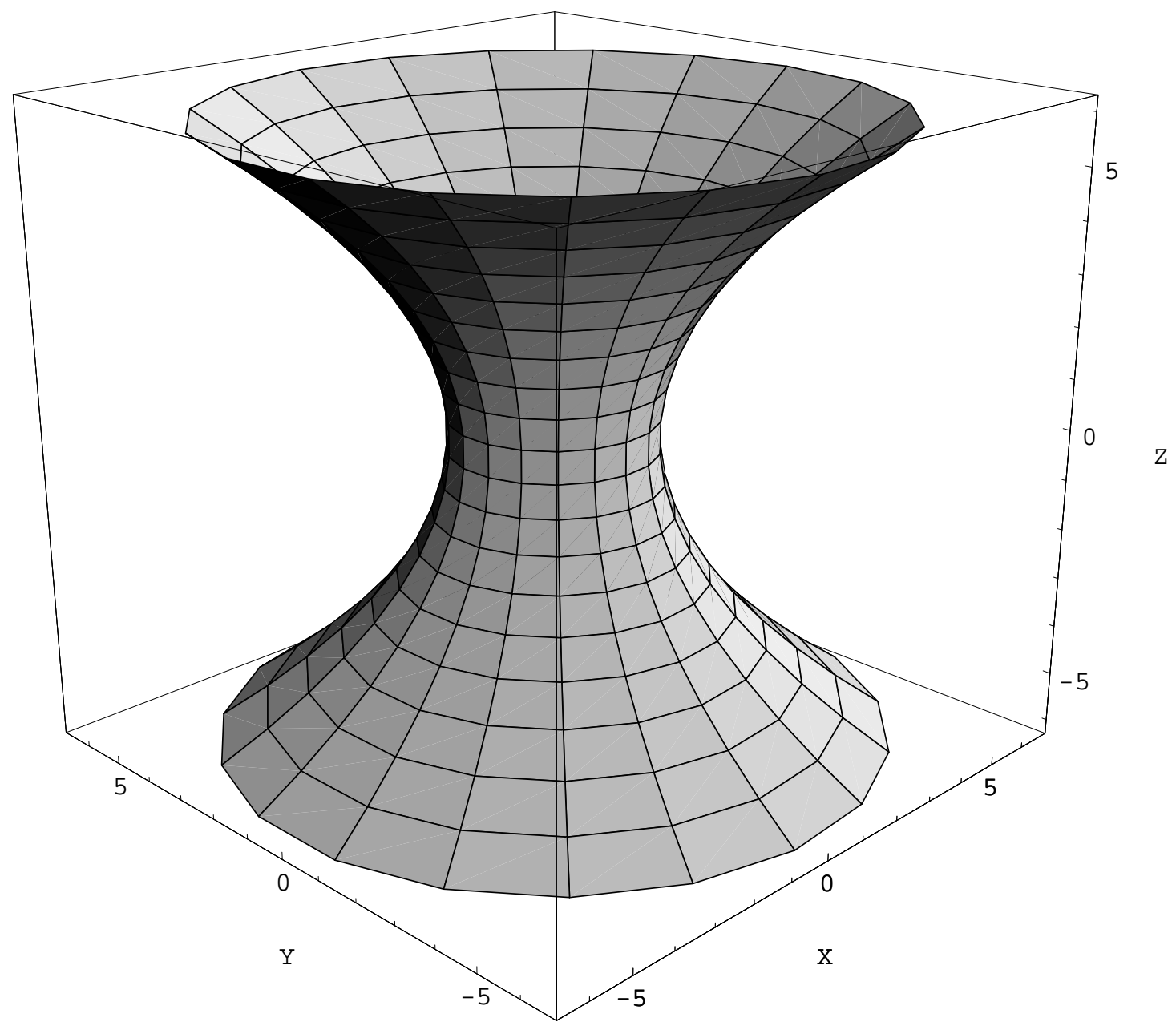

Figure 1: It shows the paraboloid of revolution for the parabola $z^{2}=8 r-16$, where $r^{2}=x^{2}+y^{2}$. 